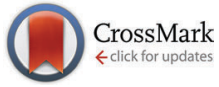

Cite this: DOI: 10.1039/c6cp01932c

\title{
Highly-oriented molecular arrangements and enhanced magnetic interactions in thin films of CoTTDPz using PTCDA templates $\dagger$
}

\author{
Keitaro Eguchi, ${ }^{a}$ Chihiro Nanjo, ${ }^{a}$ Kunio Awaga, ${ }^{\text {ab }}$ Hsiang-Han Tseng, ${ }^{c d}$ \\ Peter Robaschik ${ }^{c d}$ and Sandrine Heutz*cd
}

\begin{abstract}
In the present work, the templating effect of thin layers of perylene-3,4,9,10-tetracarboxylic dianhydride (PTCDA) on the growth of cobalt tetrakis(thiadiazole)porphyrazine (COTTDPz) thin films was examined. $X$-ray diffraction and optical absorption spectra indicate that while CoTTDPz forms amorphous thin films on the bare substrates, it forms crystalline thin films on the PTCDA templates, in which the molecular planes of COTTDPz are considered to be parallel to the substrates. Magnetic measurements reveal a significantly enhanced antiferromagnetic interaction of COTTDPz in the templated thin films, with values reaching over $13 \mathrm{~K}$. The ability to generate crystalline films and to control their orientation using molecular templates is an important strategy in the fields of organic electronics and spintronics in order to tailor the physical properties of organic thin films to suit their intended application.
\end{abstract}

Received 23rd March 2016 Accepted 9th May 2016

DOI: $10.1039 /$ c6cp01932c

www.rsc.org/pccp
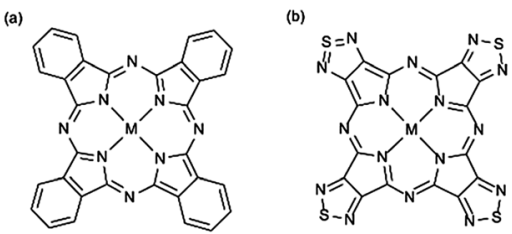

(c)

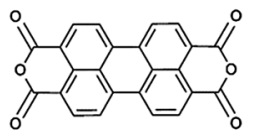
orientations and packing in the preparation of organic thin films, so as to tune their physical properties for a given application, and thereby enhance their device performance. Within these fields, metallo-phthalocyanines (MPcs; Fig. 1(a)) have been widely used due to their high thermal and chemical stability, and tunable semiconducting and magnetic properties. ${ }^{1,2}$ Their magnetic properties are strongly dependent on the metal centers and the intermolecular arrangements in the bulk crystals and thin films. There are two well-known polymorphs, the $\alpha$ - and $\beta$-forms, in the crystal structures of MPcs. Both of these consist of a 1D $\pi$ stacking of the MPc molecules, while the molecular planes make angles of $c a .27^{\circ}$ and $46^{\circ}$ with the stacking axes in the $\alpha$ - and $\beta$-forms, respectively. ${ }^{3}$ It has been shown that $\alpha$-FePc ${ }^{4}$ and $\beta-\mathrm{MnPc}^{5}$ exhibit ferromagnetic properties at a low temperature, although there are no obvious magnetic interactions in $\beta$-FePc

\footnotetext{
${ }^{a}$ Department of Chemistry, Nagoya Univeristy, Furo-cho, Chikusa-ku, 464-8602 Nagoya, Japan.E-mail: awaga@mbox.chem.nagoya-u.ac.jp

${ }^{b}$ CREST JST, Nagoya University, Furo-cho, Chikusa-ku, 464-8602, Nagoya, Japan

${ }^{c}$ Department of Materials, Imperial College London, Exhibition Road,

London SW7 2AZ, UK. E-mail: s.heutz@imperial.ac.uk

${ }^{d}$ London Centre for Nanotechnology, Imperial College London, Exhibition Road, London SW7 2AZ, UK

$\dagger$ Electronic supplementary information (ESI) available: Details of chemical synthesis, XRD data, molecular orbitals and simulated spectrum via theoretical calculations, magnetic field dependent magnetization curves. See DOI: 10.1039/c6cp01932c
}

Fig. 1 Molecular structures of metallophthalocyanine (MPc) (a), metallotetrakis(thiadiazole)porphyrazine (MTTDPz) (b), and perylene-3,4,9,10tetracarboxylic dianhydride (PTCDA) (c).

and $\alpha$-MnPc which are weakly antiferromagnetic. Furthermore, a strong antiferromagnetic interaction has been found in the powder and thin-film samples of $\alpha$-CoPc, while the $\beta$-form is paramagnetic. $^{6}$

The related metallo-tetrakis(1,2,5-thiadiazole)porphyrazines (MTTDPzs; Fig. 1(b)), which were first synthesized by Ercolani, Stuzhin and their coworkers, ${ }^{7,8}$ consist of a central metal ion and a porphyrazine ring annulated with thiadiazole rings. Although the molecular framework of MTTDPzs is similar to that of MPc, their crystal structures and electronic properties are very distinct. MTTDPzs exhibit three polymorphs, $\alpha-, \beta$ - and $\gamma$-forms, ${ }^{9}$ though there is no correspondence to the two polymorphs of MPcs. The $\alpha$-form of MTTDPz is found in $\mathrm{H}_{2}$ TTDPz, CuTTDPz and NiTTDPz, and consists of a $\pi$ stacking of 2D molecular layers, where the planar molecules are connected by side-by-side $\mathrm{S}^{\delta+} \ldots \mathrm{N}^{\delta-}$ electrostatic interactions within the layers. The $\beta$-form is found in FeTTDPz, CoTTDPz and ZnTTDPz, and consists of a 1D zigzag chain, formed by a coordination bonding between the metal ion and the thiadiazole ring. The $\gamma$-form is found in 
CuTTDPz and NiTTDPz, and consists of a 1D $\pi$ stacking of the molecules, which is similar to that in the $\beta$-form of MPc, although in the former, two neighboring stacking columns form a ladder-type structure with interchain $S^{\delta+} \cdots \mathrm{N}^{\delta-}$ contacts. In these polymorphs of MTTDPzs, $\beta$-CoTTDPz and $\beta$-FeTTDPz exhibit strong antiferromagnetic interactions with $2 J / k_{\mathrm{B}}=-17.8$ and $-9.1 \mathrm{~K}$, respectively, caused by a dimerized structure, which is formed in the interchain arrangement of the $\beta$-form. ${ }^{9}$ VOTTDPz crystallizes into two polymorphs, which are very different from those of the other MTTDPzs, and exhibits weak ferromagnetic interactions due to the negative spin density on the oxygen atom. ${ }^{10}$ Furthermore, the MTTDPz family has been shown to exhibit electron-accepting properties and n-type conduction due to the presence of the electronegative thiadiazole rings, in contrast to most of the MPcs, which are electron donors with p-type semiconductive behavior. ${ }^{10-12}$

In addition to studies on the bulk crystals of MTTDPzs, we have investigated their thin-film formation on various substrates, such as glass, $\mathrm{Si}$ wafer and metal films. Although $\mathrm{H}_{2}$ TTDPz formed crystalline films with a flat-lying structure, ${ }^{13}$ which is the same as that found in the $\alpha$-form crystal structure, the other derivatives formed amorphous films, presumably due to competition among the various structure-controlling factors, such as $\pi-\pi$ interaction, $\mathrm{S}^{\delta+} \ldots \mathrm{N}^{\delta-}$ electrostatic interaction and coordination bonding. Molecular templating is an attractive method for improving or modifying the crystalline order in molecular thin films. ${ }^{14}$ Recently, Serri and coworkers reported a template method for controlling the molecular orientations of MPcs in their thin films and reached magnetic couplings at around $100 \mathrm{~K} .{ }^{6}$ Specifically, CoPc was found to form thin films with a flat-lying structure on thin template layers of perylene-3,4,9,10tetracarboxylic dianhydride (PTCDA; Fig. 1(c)) due to $\pi-\pi$ interactions between CoPc and PTCDA at the interface. However, without this PTCDA template, CoPc formed thin films with a standing structure, in which molecular planes were nearly perpendicular to the substrates. ${ }^{6}$ In the present study, we prepared films of COTTDPz with and without a pre-deposited PTCDA template, and examined its effect on their structure, optical absorption and magnetic properties.

\section{Experimental}

CoTTDPz was prepared, using a method similar to that used in the literature (see the ESI $\dagger)^{7,8}$ The obtained CoTTDPz was purified by vacuum sublimation with a continuous $\mathrm{N}_{2}$ gas flow $\left(40-50 \mathrm{ml} \mathrm{min}^{-1}\right)$. Commercial PTCDA (purity $>98 \%$ ) was used without further purification. For thin film preparations, CoTTDPz and PTCDA were mounted in the Knudsen cells for molecular deposition, and were degassed for $\sim 3$ hours before deposition. Polyimide films (25 $\mu \mathrm{m}$ in thickness) and glass plates (1 $\mathrm{mm}$ in thickness) were used as substrates. Prior to deposition, the substrates were cleaned in an ultrasonic bath with acetone and propan-2-ol, and then placed in a deposition chamber (SPECTROS organic molecular beam deposition chamber from Kurt J. Lesker with a base pressure of $10^{-5} \mathrm{~Pa}$ ).
The PTCDA templates were prepared by molecular deposition on the clean substrates. The deposition rate was controlled at $\sim 0.2 \AA \mathrm{s}^{-1}$ using a quartz crystal microbalance (QCM) monitor near the sample holder. CoTTDPz was deposited on the bare and PTCDA-templated substrates at room temperature with a deposition rate of $0.1-0.03 \AA \mathrm{s}^{-1}$. The thin-film thicknesses were set to be 20 and $100 \mathrm{~nm}$ for PTCDA and CoTTDPz, respectively. During all the depositions, the pressure was maintained at less than $4.5 \times 10^{-5} \mathrm{~Pa}$.

Structural and morphological characterization was performed at room temperature using atomic force microscopy (AFM; Seiko Instruments SPI 3800/SPA 400), scanning electron microscopy (SEM; Hitachi SU 6600), and powdered X-ray diffraction (XRD; PANalytical X-pert Powder diffractometer). The AFM images were obtained in dynamic force mode under ambient pressure using a $\mathrm{Si}_{3} \mathrm{~N}_{4}$ tip. The SEM measurements were carried out for the observation of cross-sectional images of the films under high vacuum conditions $\left(P<10^{-3} \mathrm{~Pa}\right)$ with an incident electron energy of $1.5 \mathrm{keV}$. The XRD signals were recorded using $\mathrm{Cu} \mathrm{K} \alpha$ radiation $(40 \mathrm{kV}, 40 \mathrm{~mA}$, nickel filter) and the Bragg-Brentano geometry operated in the $\theta-2 \theta$ mode.

Optical absorption of the CoTTDPz films was measured using an ultraviolet-visible (UV-vis) photospectrometer (Perkin Elmer Lambda 25 spectrometer) in transmission mode at $300 \mathrm{~K}$. The data were used without correction for reflections at the interfaces. The solution UV-vis absorption spectra of CoTTDPz in a mixture of $\mathrm{H}_{2} \mathrm{SO}_{4}$ and $N, N$-dimethylformamide (DMF) were also measured for comparison with the films. The magnetic properties of the CoTTDPz films were examined using a superconducting quantum interference device (SQUID) magnetometer (Quantum Design MPMS-XL magnetometer). The temperaturedependent magnetizations were measured in the temperature range of 2-300 K under the magnetic field of $0.5 \mathrm{~T}$ parallel to the films.

Molecular orbital calculations for CoTTDPz were performed using the Gaussian09 package ${ }^{15}$ using DFT-UB3LYP method and $6-311 \mathrm{G}+(\mathrm{d})$ as a basis set. The molecular structure of CoTTDPz was optimized in the $D_{4 \mathrm{~h}}$ symmetry (Fig. S2, S3 and Table S1, ESI $\dagger$ ), and no imaginary frequencies were obtained in the optimized geometry. A simulation of the electronic transition spectrum of CoTTDPz was obtained from a time-dependent DFT calculation (Fig. S4 and Table S2, ESI $\dagger$ ).

\section{Results and discussion}

\subsection{Structural characterization of CoTTDPz films}

Recently, PTCDA thin films have been found to operate as an excellent template to control the molecular orientations of thin films deposited onto them. ${ }^{6}$ This molecule exhibits a highly oriented structure when deposited at room temperature on inactive insulators such as glass, making the (102) plane in the $\alpha$ polymorph parallel to the substrates. ${ }^{16-18}$ In this arrangement, the molecular planes are nearly parallel to the substrate, making their $\pi$ orbitals normal to them. ${ }^{19,20}$ Therefore, $\pi$-conjugated molecules, such as MPcs, can be adsorbed with a flat-lying orientation via $\pi-\pi$ interactions at the interface. ${ }^{6,17,18,21,22}$ 

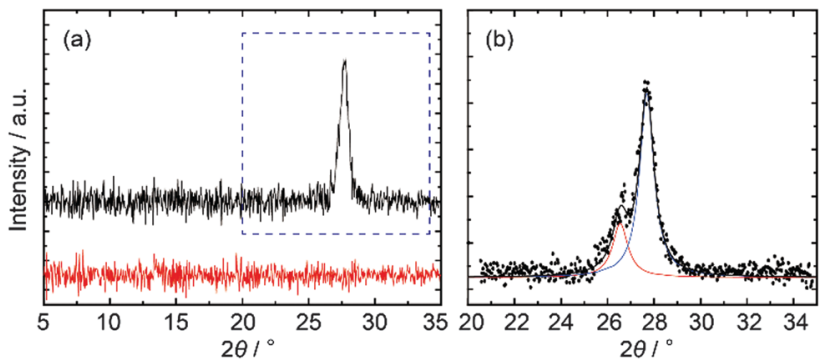

Fig. 2 (a) XRD patterns of CoTTDPz (100 nm)/polyimide (red) and CoTTDPz (100 nm)/PTCDA (20 nm)/polyimide (black). (b) High resolution XRD pattern of CoTTDPz $(100 \mathrm{~nm}) /$ PTCDA $(20 \mathrm{~nm}) /$ polyimide in the $2 \theta$ range of $20-35^{\circ}$. The red and blue curves indicate the results of the peak decomposition, using the Lorentzian functions.

To elucidate the template effect of PTCDA on the deposition of CoTTDPz, we prepared $100 \mathrm{~nm}$ thick CoTTDPz films on polyimide and glass substrates, with and without a $20 \mathrm{~nm}$ thick PTCDA layer previously deposited onto the substrate, and use the following nomenclature: CoTTDPz (100 nm)/PTCDA (20 or $0 \mathrm{~nm}) / \mathrm{glass}$ or polyimide.

Fig. 2(a) shows the XRD patterns of $100 \mathrm{~nm}$ thick CoTTDPz films on polyimide with (black) and without (red) a $20 \mathrm{~nm}$ thick PTCDA templating layer. In the absence of PTCDA, no peak can be observed in the whole $2 \theta$ range of $5-35^{\circ}$, indicating that CoTTDPz does not form crystalline films on polyimide, or that the crystal sizes are below the threshold of detection of our labbased diffractometer. However, when deposited on a template layer of PTCDA, a strong peak is observed at $2 \theta=28^{\circ}$. Fig. 2(b) shows the higher resolution of this peak, which clearly demonstrates that the strong peak at $2 \theta=28^{\circ}$ consists of two contributions. The peak analysis, assuming Lorentzian curves, concludes the presence of a weaker peak at $26.5^{\circ}$ (red curve) and a stronger peak at $27.7^{\circ}$ (blue curve), which correspond to interplanar distances, $d$, of $3.6 \AA$ and $3.3 \AA$, respectively. The average crystallite size is roughly estimated as $13 \mathrm{~nm}$ from the full widths at half maxima of these peaks by using the Scherrer formula with a Scherrer constant of 0.9. ${ }^{23}$ The diffraction peaks of CoTTDPz on PTCDA do not correspond to any prominent peaks in the $\beta$-CoTTDPz structure. However, by comparison with the other polymorphic forms of the TTDPz series, ${ }^{9}$ the region around $2 \theta=27^{\circ}$ shows the strong diffractions from the $(10-3)$ plane in the $\alpha$ form with $2 \theta=27.2^{\circ}$ and, the (142) and (133) planes in the $\gamma$ form with $2 \theta=27.0^{\circ}$ and $27.3^{\circ}$, respectively, all corresponding to orientations which are nearly parallel to the molecular planes (see Fig. S1, ESI $\dagger$ ). Interestingly, both these polymorphs display molecular columns dominated by $\pi-\pi$ interactions, rather than the dimers characterizing the $\beta$ form. In particular, the $\alpha$ phase arranges in molecular layers characterized by strong intermolecular $\mathrm{S} \cdots \mathrm{N}$ interactions, and separated by approximately $3.3 \AA$. These layers can effectively interact via $\pi-\pi$ interactions with the PTCDA film whose molecules are lying nearly flat on the substrate, as previously observed for the MPcs. ${ }^{6,17,18,21,22}$ This gives rise to a diffraction at approximately $2 \theta=27.3^{\circ}(d=3.3 \AA)$, as previously observed in the $\mathrm{H}_{2}$ TTDPz films. ${ }^{13}$ This templating effect was not limited to polyimide substrates, as a similar effect was observed for films deposited on glass (see Fig. S1, ESI $\dagger$ ).

The surface and internal morphologies of the films on polyimide were investigated using AFM and SEM. Fig. 3(a) and (b) show the AFM images for the surfaces of a bare polyimide film and CoTTDPz (100 nm)/polyimide, respectively. The former shows small needle-like features superimposed onto large-scale roughness, and the latter also shows a rough surface. Since their height differences, shown in the bottom panels of Fig. 3(a) and (b), are similar, the surface roughness of the CoTTDPz is considered to reflect that of the polyimide underlayer. Fig. 3(c) shows the SEM image for a cross-section of CoTTDPz $(100 \mathrm{~nm}) /$ polyimide,
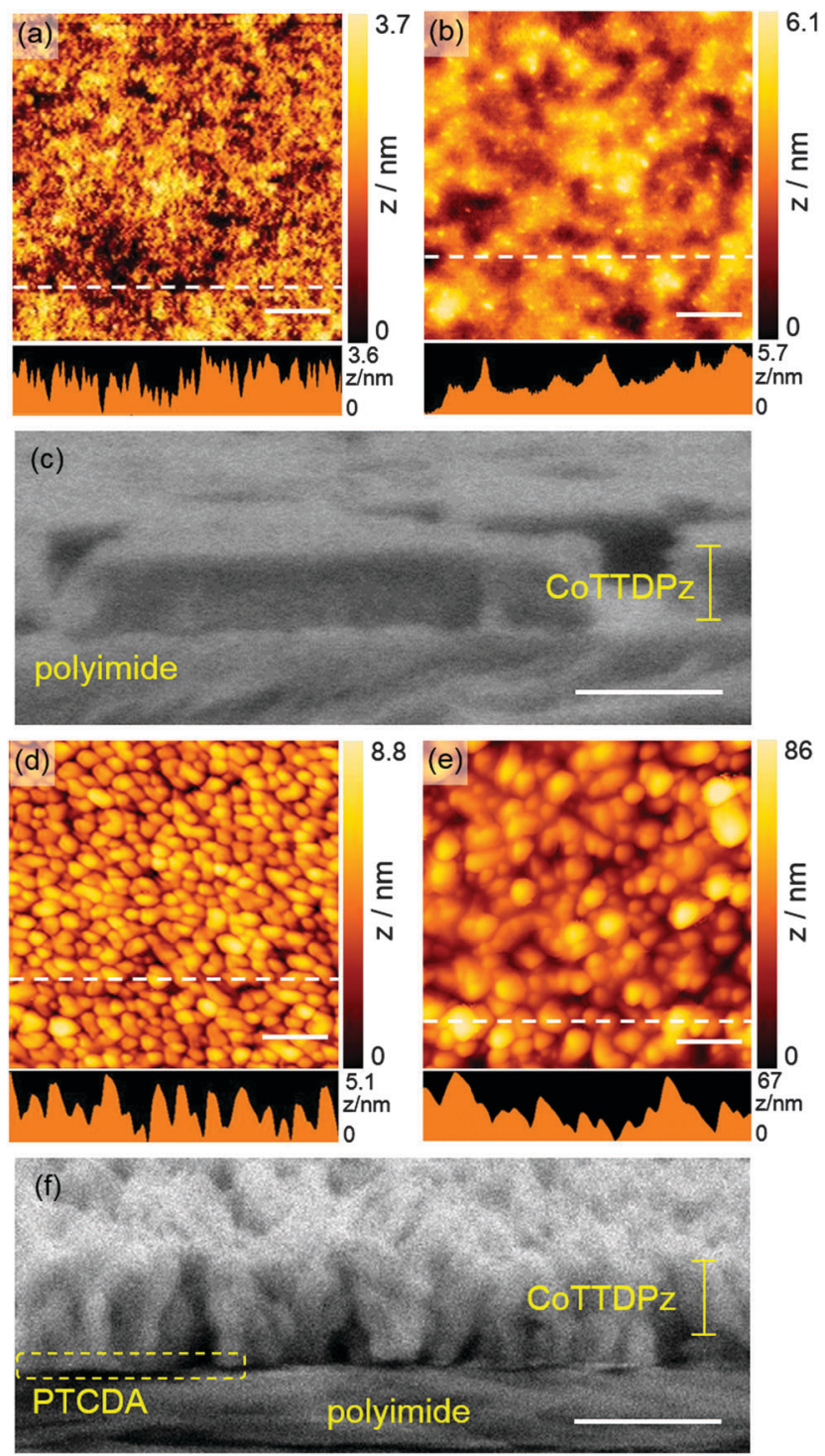

Fig. 3 AFM images taken in dynamic force mode for a bare polyimide film (a) and CoTTDPz $(100 \mathrm{~nm}) /$ polyimide (b). The lower parts of these panels show the cross-sectional line profiles along the broken lines in the upper parts. SEM image of a cross-section of COTTDPz $(100 \mathrm{~nm}) /$ polyimide (c). AFM images of PTCDA $(20 \mathrm{~nm}) /$ polyimide (d) and CoTTDPz $(100 \mathrm{~nm}) /$ PTCDA $(20 \mathrm{~nm}) /$ polyimide (e). SEM image of a cross-section of CoTTDPz $(100 \mathrm{~nm}) /$ PTCDA $(20 \mathrm{~nm}) /$ polyimide (f). The white and yellow bars indicate distances of 200 and $100 \mathrm{~nm}$, respectively. 
indicating several smooth-surface grains with nearly the same height. Fig. 3(d) and (e) show the AFM images for the surfaces of PTCDA (20 nm)/polyimide and CoTTDPz (100 nm)/PTCDA (20 nm)/polyimide, respectively. The former shows smooth grains with a diameter of $50 \mathrm{~nm}$ and a height scale of $5 \mathrm{~nm}$ on the line scan. The latter also consists of smooth grains with diameters of 50-120 nm, but its height difference exceeds that of the former by one order of magnitude. Fig. 3(f) shows the SEM image for a cross-section of the template CoTTDPz, in which the CoTTDPz layer resembles an assembly of nanocrystals grown normal to the substrate. This is consistent with the enhanced height difference found in the AFM image.

It is concluded that, although the templating effects of the PTCDA films are known for MPcs, ${ }^{6,17,18,21,22}$ in which the $\pi-\pi$ interactions dominate the growth, the PTCDA templates are also very effective for CoTTDPz films, in which $\pi-\pi$ interactions are less dominant, due to competition with coordination bonding and $\mathrm{S}^{\delta+} \cdots \mathrm{N}^{\delta-}$ electrostatic interactions. In addition, the templated molecular arrangement of CoTTDPz is found to result in crystalline films with the long axis of the crystal aligned normal to the substrates.

\subsection{Optical properties}

The presence of a PTCDA template as mentioned above yields a significant difference in the structure of thin films of CoTTDPz, compared to template-free growth. This is expected to result in a significant difference between their physical properties. Fig. S4 in the ESI $\dagger$ shows the UV-vis absorption spectrum of CoTTPDz, dissolved in a mixture of concentrated $\mathrm{H}_{2} \mathrm{SO}_{4}$ and DMF, and the results of the DFT calculation. The observed absorptions can be well explained by the simulated optical transitions, on condition that the calculated transition energies are shifted by $0.155 \mathrm{eV}$ toward the lower energy side. The absorption peak at $2.0 \mathrm{eV}$, associated with a vibronic structure, is the Q-band, which corresponds to the HOMO-LUMO transition, and the peaks at around $4.0 \mathrm{eV}$ are the Soret-band.

Fig. 4(a) shows the optical absorption spectra on glass substrates of CoTTDPz (100 nm)/glass (black), CoTTDPz (100 nm)/PTCDA $(20 \mathrm{~nm}) /$ glass (red), and PTCDA (20 nm)/glass (blue). Compared with the solution spectrum in Fig. S4 (see the ESI $\dagger$ ), the onsets of the Q-bands are shifted toward the lower energy side, although the Soret-bands appear at nearly the same energy. Fig. 4(b) shows the absorptions of the same thin films on polyimide substrates (color-coding persists). Although the Soret-bands are masked by the polyimide absorption, the Q-bands of these thin films exhibit the same feature as found in the thin films on glass. In the absorption range of the Q-band, all CoTTDPz films exhibit three distinct transitions, denoted as A-C in Fig. 4. Peak D observed in the template films is assignable to the absorption of PTCDA, since it also appears in the absorption spectra of the pure PTCDA films. The other peaks (A-C) are ascribed to the vibronic structure of the Q-band of CoTTDPz, because the energy separation between them $(c a .0 .20 \mathrm{eV})$ is rather close to that for the vibronic structure in the solution absorption spectrum (0.19 eV, see Fig. S4, ESI $\dagger$ ). A characteristic PTCDAtemplate effect can be observed in the intensity ratio between

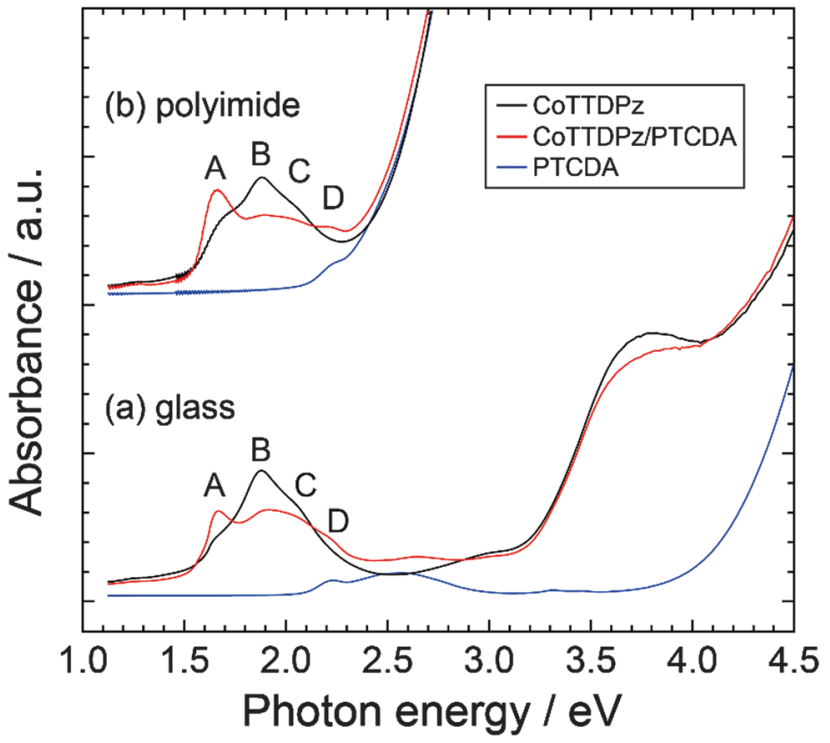

Fig. 4 (a) UV-vis absorption spectra of films of COTTDPz (100 nm) (black), COTTDPz (100 nm)/PTCDA (20 nm) (red) and PTCDA (20 nm) (blue) on glass substrates. (b) Same films and color coding on polyimide substrates.

the A and B bands; the A band is much stronger than B for the templated films with well-oriented molecular arrangements of CoTTDPz caused by PTCDA, while the B band is the strongest for the amorphous films of the non-templated samples. This might suggest that band A is caused by an intermolecular effect. In any case, it can be concluded that the well-oriented and crystallised CoTTDPz on PTCDA induced the characteristic intensity increase of band $\mathrm{A}$.

\subsection{Magnetic properties}

The magnetic properties of the CoTTDPz thin films with and without the PTCDA template were examined to elucidate the template effect on the electronic structure of CoTTDPz. The inset of Fig. 5 schematically shows the sample preparation for the magnetic measurements. Belt-shaped samples (ca. $80 \mathrm{~mm}$ $\times 4 \mathrm{~mm}$ ) of CoTTDPz (100 nm), with and without PTCDA $(20 \mathrm{~nm})$, were fabricated on polyimide films, in which the amount of CoTTDPz was $\mathrm{ca} \cdot 10^{-7} \mathrm{~mol}$. The polyimide films were rolled and then inserted into the straws for the SQUID measurements. $^{22}$ Fig. 5 shows a comparison between the temperature dependences of the paramagnetic susceptibilities $\chi_{\mathrm{p}}$ for the two samples, measured under an external magnetic field of $0.5 \mathrm{~T}$. The diamagnetic contributions from the polyimide films and straws were eliminated due to their homogeneity in the scanning region. ${ }^{22}$ We used the literature data ${ }^{24}$ for the diamagnetic corrections of the framework and subtracted them from the experimental data. The small diamagnetic contribution of PTCDA, estimated from that of perylene, ${ }^{25}$ was also subtracted for the template sample. The $\chi_{\mathrm{p}}$ values of non-templated sample monotonically increase with a decrease in temperature, and the data in the range of $20-300 \mathrm{~K}$ can be fitted to the CurieWeiss law, $\chi_{\mathrm{p}}=C /(T-\theta)$, with a Curie constant of $C=0.71 \pm$ $0.10 \mu_{\mathrm{B}} \mathrm{K} \mathrm{T}^{-1}\left(=0.39 \pm 0.06 \mathrm{emu} \mathrm{K} \mathrm{mol}{ }^{-1}\right)$, and a Weiss constant 


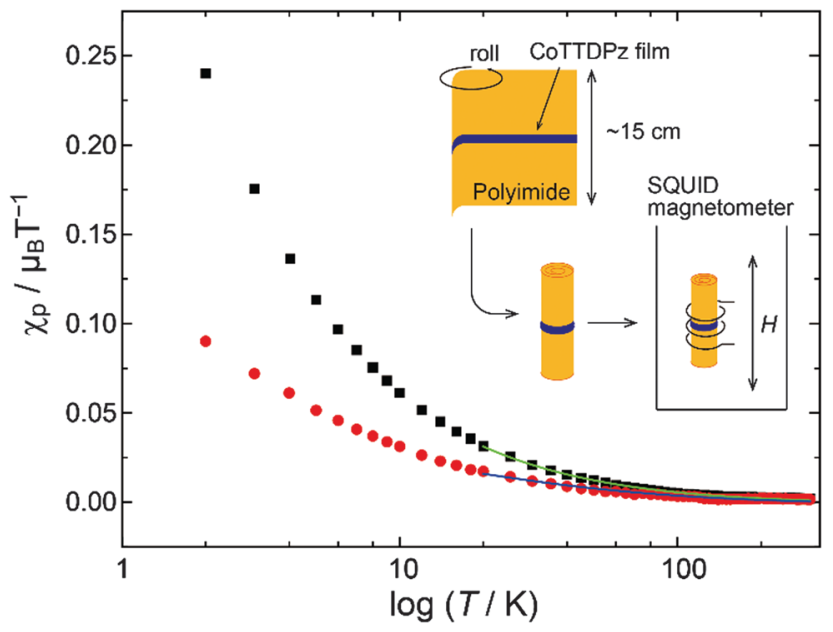

Fig. 5 Temperature dependence of the paramagnetic susceptibilities of CoTTDPz $(100 \mathrm{~nm}) /$ polyimide (black) and CoTTDPz (100 nm)/PTCDA $(20 \mathrm{~nm}) /$ polyimide $(\mathrm{red})$. The green and red curves show the theoretical best fits of the Curie-Weiss law (see the main text). The inset schematically shows the sample preparation for the magnetic measurements on polyimide substrates.

of $\theta=-1.7 \pm 1.2 \mathrm{~K}$ (green). The large errors were caused by an ambiguity in the sample weights, and the small signal intensities at high temperatures. The templated film also exhibits a similar behavior, but the $\chi_{\mathrm{p}}$ values are much more suppressed, especially at low temperatures; this dependence can be also fitted to the Curie Weiss law with $C=0.59 \pm 0.10 \mu_{\mathrm{B}} \mathrm{K} \mathrm{T}^{-1}(=0.33 \pm$ $0.06 \mathrm{emu} \mathrm{K} \mathrm{mol}{ }^{-1}$ ), $\theta=-13.6 \pm 4.4 \mathrm{~K}$ (blue), indicating a significant enhancement of the intermolecular antiferromagnetic interactions. This must be caused by the well-oriented structure in the templated film. Although the zero-field-cooled magnetizations of these two films were also measured, there was little difference from those under the field-cooled conditions (see Fig. S5, ESI $\dagger$ ). The magnetic field dependence of the magnetizations of these two CoTTDPz films was also examined at the temperatures of $2,7,12,25$, and $50 \mathrm{~K}$. The results are shown in Fig. S6 (see the ESI $\dagger$ ), where the magnetizations are plotted as a function of $\mu_{0} H / T$. These results are consistent with the results of the temperature dependence of $\chi_{\mathrm{p}}$. It is worth noting that the observed antiferromagnetic interaction $(\theta=-13 \mathrm{~K})$ of CoTTDPz $(100 \mathrm{~nm}) /$ PTCDA $(20 \mathrm{~nm}) /$ polyimide with high crystallinity is comparable to that of $2 J / k_{\mathrm{B}}=-17.8 \mathrm{~K}^{9}$ in the bulk crystals of CoTTDPz, where CoTTDPz forms a face-to-face dimer with an interplanar distance of $3.4 \AA$.

\section{Conclusions}

We examined the template effect of PTCDA on CoTTDPz thin films. While the thin films of CoTTDPz were amorphous without this template, it formed crystalline thin-films with a flatlying molecular arrangement when deposited on the template, which was probably triggered by $\pi-\pi$ interactions with the PTCDA. To our knowledge, this is the first example of PTCDA promoting crystallinity in an amorphous film. The AFM and
SEM measurements of the CoTTDPz/PTCDA films revealed that the CoTTDPz layer consisted of nanocrystals, which had grown normal to the substrates. These structural features of the templated thin films resulted in a characteristic shape of the Q-band in the optical absorption spectra, and in the enhanced antiferromagnetic interaction in the temperature dependence of the magnetic susceptibility.

\section{Acknowledgements}

The authors would like to thank Mr K. Matsushita, Mr T. Komoto, and Mr R. Sasa for their technical support in preparing the sample holders and the QCM monitor. This work was supported by the Japan Society for the Promotion of Science (JSPS) Core-to-Core Program, Advanced Research Networks and JSPS KAKENHI Grant Number 15J11122. A part of this work was conducted at the Institute for Molecular Science (IMS), with support from the Nanotechnology Platform Program (Molecule and Material Synthesis) of the Ministry of Education, Culture, Sports, Science and Technology (MEXT), Japan.

\section{Notes and references}

1 F. H. Moser and A. L. Thomas, The Phthalocyanines: Properties, CRC press, The United States, 1983.

2 F. H. Moser and A. L. Thomas, The Phthalocyanines: Manufacture and Applications, CRC press, The United States, 1983.

3 K. Y. Law, Chem. Rev., 1993, 93, 449-486.

4 M. Evangelisti, J. Bartolomé, L. de Jongh and G. Filoti, Phys. Rev. B: Condens. Matter Mater. Phys., 2002, 66, 144410.

5 C. G. Barraclough, J. Chem. Phys., 1970, 53, 1638.

6 M. Serri, W. Wu, L. R. Fleet, N. M. Harrison, C. F. Hirjibehedin, C. W. Kay, A. J. Fisher, G. Aeppli and S. Heutz, Nat. Commun., 2014, 5, 3079.

7 E. M. Bauer, D. Cardarilli, C. Ercolani, P. A. Stuzhin and U. Russo, Inorg. Chem., 1999, 38, 6114-6120.

8 P. A. Stuzhin, E. M. Bauer and C. Ercolani, Inorg. Chem., 1998, 37, 1533-1539.

9 Y. Suzuki, M. Fujimori, H. Yoshikawa and K. Awaga, Chem. Eur. J., 2004, 10, 5158-5164.

10 Y. Miyoshi, K. Takahashi, T. Fujimoto, H. Yoshikawa, M. M. Matsushita, Y. Ouchi, M. Kepenekian, V. Robert, M. P. Donzello, C. Ercolani and K. Awaga, Inorg. Chem., 2011, 51, 456-462.

11 Y. Miyoshi, T. Fujimoto, H. Yoshikawa, M. M. Matsushita, K. Awaga, T. Yamada and H. Ito, Org. Electron., 2011, 12, 239-243.

12 C. Nanjo, T. Fujimoto, M. M. Matsushita and K. Awaga, J. Phys. Chem. C, 2014, 118, 14142-14149.

13 Y. Miyoshi, M. Kubo, T. Fujinawa, Y. Suzuki, H. Yoshikawa and K. Awaga, Angew. Chem., Int. Ed., 2007, 46, 5532-5536.

14 J. Yang, D. Yan and T. S. Jones, Chem. Rev., 2015, 115, 5570-5603.

15 M. J. Frisch, G. W. Trucks, H. B. Schlegel, G. E. Scuseria, M. A. Robb, J. R. Cheeseman, G. Scalmani, V. Barone, 
B. Mennucci, G. A. Petersson, H. Nakatsuji, M. Caricato, X. Li, H. P. Hratchian, A. F. Izmaylov, J. Bloino, G. Zheng, J. L. Sonnenberg, M. Hada, M. Ehara, K. Toyota, R. Fukuda, J. Hasegawa, M. Ishida, T. Nakajima, Y. Honda, O. Kitao, H. Nakai, T. Vreven, J. A. Montgomery Jr, J. E. Peralta, F. Ogliaro, M. J. Bearpark, J. Heyd, E. N. Brothers, K. N. Kudin, V. N. Staroverov, R. Kobayashi, J. Normand, K. Raghavachari, A. P. Rendell, J. C. Burant, S. S. Iyengar, J. Tomasi, M. Cossi, N. Rega, N. J. Millam, M. Klene, J. E. Knox, J. B. Cross, V. Bakken, C. Adamo, J. Jaramillo, R. Gomperts, R. E. Stratmann, O. Yazyev, A. J. Austin, R. Cammi, C. Pomelli, J. W. Ochterski, R. L. Martin, K. Morokuma, V. G. Zakrzewski, G. A. Voth, P. Salvador, J. J. Dannenberg, S. Dapprich, A. D. Daniels, Ö. Farkas, J. B. Foresman, J. V. Ortiz, J. Cioslowski and D. J. Fox, Gaussian, Inc., Wallingford CT, 2009.

16 S. Heutz, A. J. Ferguson, G. Rumbles and T. S. Jones, Org. Electron., 2002, 3, 119-127.
17 S. Heutz, R. Cloots and T. S. Jones, Appl. Phys. Lett., 2000, 77, 3938-3940.

18 P. Sullivan, T. S. Jones, A. J. Ferguson and S. Heutz, Appl. Phys. Lett., 2007, 91, 233114.

19 T. Ogawa, K. Kuwamoto, S. Isoda, T. Kobayashi and N. Karl, Acta Crystallogr., Sect. B: Struct. Sci., 1999, 55, 123-130.

20 H.-H. Tseng, M. Serri, N. M. Harrison and S. Heutz, J. Mater. Chem. C, 2015, 3, 8694-8699.

21 O. D. Gordan, T. Sakurai, M. Friedrich, K. Akimoto and D. R. T. Zahn, Org. Electron., 2006, 7, 521-527.

22 S. Heutz, C. Mitra, W. Wu, A. J. Fisher, A. Kerridge, M. Stoneham, A. H. Harker, J. Gardener, H. H. Tseng, T. S. Jones, C. Renner and G. Aeppli, Adv. Mater., 2007, 19, 3618-3622.

23 J. I. Langford and A. J. C. Wilson, J. Appl. Crystallogr., 1978, 11, 102-113.

24 C. G. Barraclough, J. Chem. Phys., 1971, 55, 1426.

25 H. Shiba and G. Hazato, Bull. Chem. Soc. Jpn., 1949, 22, 92-96. 